\title{
DISEÑO Y CONSTRUCCIÓN DE UN ROBOT BÍPEDO EXPERIMENTAL
}

\author{
José Larriva, Edyson Guillermo, José Trelles, Oscar Vele*
}

\section{Antecedentes}

En la actualidad nuevas tecnologías y ramas de la investigación han volcado su atención hacia la comunidad científica; entre ellas tenemos a la genética, la exploración del espacio, la robótica, etc., todas éstas con grandes e importantes aplicaciones, pero con una complejidad que requiere de la participación de una mayor cantidad y calidad de científicos en todo el mundo.

El presente proyecto pertenece al campo de la robótica, rama que no ha sido desarrollada en nuestro país; por ello mediante la realización de un robot bípedo (robot preparado y construido para que pueda movilizarse en dos piernas simulando la caminata de una persona) se pretende sentar un primer escalón sobre el cual se levanten proyectos de mayor complejidad y aplicación en este campo.

Existen grandes avances en todo el mundo en el desarrollo de robots bípedos y humanoides (robots con apariencia humana), tal es el caso de Honda al construir a ASIMO su robot; Sony, con su robot Qrio; etc., de aquí que resulta ilusorio pensar que los autores de este proyecto pretendan rivalizar con estas grandes compañías que han dedicado años a la investigación y creación de éstos prototipos. Elementos y condiciones físicas como económicas son factores decisivos; además, que son empresas que han dedicado años a la investigación tecnológica para desarrollar esta clase de robots, pero como se mencionó anteriormente, este proyecto intenta ser la base sobre la cual despeguen otros.

La pregunta podría ser: ¿Por qué robots humanoides?

La respuesta está fundamentada en los siguientes dos aspectos:

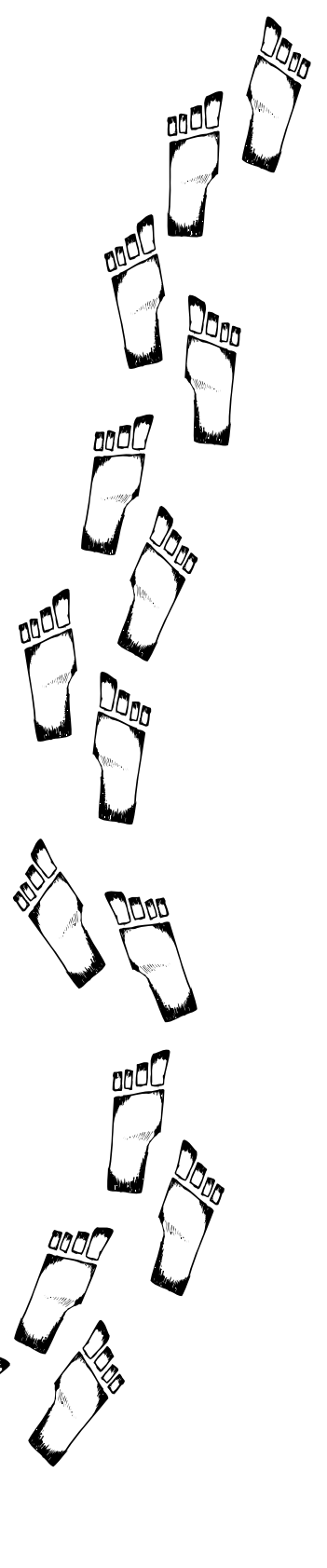


- Generación de campos de investigación tales como: Mecatrónica, Teoría de Control, Integración y Fusión sensorial, Simulación computarizada, etc.

- Aplicaciones en diferentes campos como: publicidad, entretenimiento, inclusive en un futuro cercano podríamos tener asistentes domésticos robots, robots humanoides para la exploración del espacio o para ambientes peligrosos, etc.

La principal ventaja de los robots humanoides sobre otro tipo de ro- bots es que pueden desenvolverse en superficies irregulares, gradas, etc., sin embargo, la complejidad en el diseño y control aumenta considerablemente.

A partir de lo dicho anteriormente, se puede notar que el estudio de robots bípedos es un tema apasionante con amplios campos de investigación y aplicación, la importancia de COMENZAR en nuestro medio con la realización de este tipo de proyectos.

Se ha realizado el diseño y construcción de un robot bípedo en su

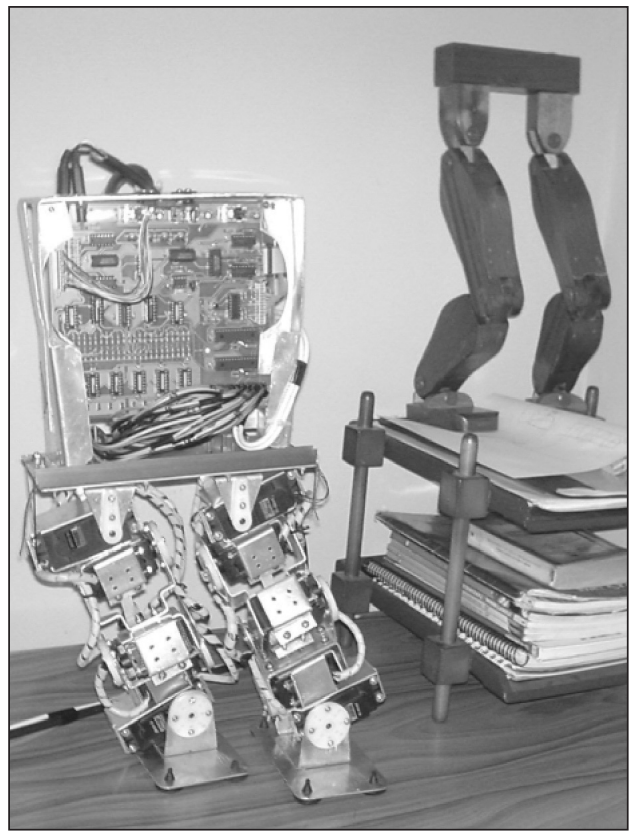

Foto 1

Robot en caminata 
primera fase, es decir, un robot que tiene la capacidad de caminar en línea recta sin perder el equilibrio.

\section{Fundamentos de la caminata} bípeda

Los humanos utilizamos muy poca energía (actividad muscular) y un mínimo de procesamiento por parte del cerebro para mantener una caminata a un ritmo moderado, sin em- bargo, el proceso de caminar es mucho más complejo de lo que parece, ya que no sólo se requiere de "dar pasos"; pues en este proceso están involucrados pies, cadera, torso, brazos, hombros, cabeza, etc. Una coordinación perfecta de todos los elementos anteriores hace que la caminata humana sea eficiente y al mismo tiempo "elegante".

En la siguiente figura se observa un medio ciclo en la caminata humana:

Foto 2

Medio ciclo de caminata humana

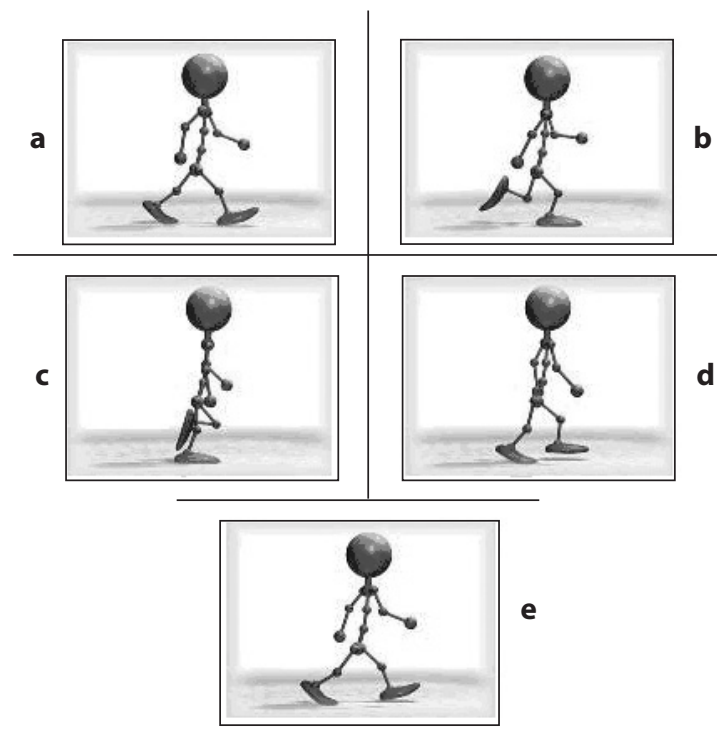


La caminata comienza con los dos pies extendidos y sobre el suelo (figura a), en donde el equilibrio no es muy significativo. El gran problema comienza al levantar uno de los dos pies para realizar los movimientos de las figuras b, c y d, ya que la tendencia es caer hacia ese lado y hacia delante o atrás dependiendo del estado dinámico del robot en ese instante. Para evitar que el robot caiga se deben realizar correctivos a los movimientos del robot (p. ej.: mover el centro de masa al lado contrario al pie levantado), permitiendo así la estabilidad dinámica de la caminata. El medio ciclo se completa cuando los dos pies vuelven a estar en el suelo (figura e). El otro medio ciclo es idéntico, solamente que el pie que se levanta es el que anteriormente servía de apoyo y viceversa.

\section{Simulación del robot}

Al considerar la dificultad para realizar un modelo mecánico del robot en forma inmediata, fue necesario buscar una herramienta que permita simular cuerpos en movimiento.

En cualquier proceso en el que se involucren partes mecánicas en movimiento se impone un exhaustivo análisis físico y matemático tomando en cuenta la dificultad que eso conlleva. Otra herramienta de análisis para estos fenómenos son los programas de simulación, los cuales existen de todos los tipos y para muchas aplicaciones; por mencionar algunos se tiene a MATLAB, VisSim, ThreeDimSim, etc., los mismos que facilitan de gran forma el análisis de sistemas mecánicos en movimiento, algunos de ellos incluso trabajan con entornos en tres dimensiones. La ventaja de utilizar estos programas de simulación es que se optimiza el análisis matemático y en muchos de los casos se pueden obtener importantes deducciones en forma eficaz y rápida. Otra ventaja de los programas de simulación es que su complejidad en el diseño y análisis no aumenta a medida que se adicionan componentes, cosa que no sucede con el análisis matemático. Se ha mencionado que la simulación sustituye de cierta forma al análisis físico-matemático, pero cabe destacar que para realizar una simulación que represente de buena forma a la realidad se deben tener muy claros los conceptos y principios fundamentales de la física. Es importante destacar que ningún software emulará en forma efectiva a un fenómeno físico, de aquí, que la pericia, conocimiento y sentido común del programador juegan un papel fundamental.

La simulación del robot fue realizada en MATLAB mediante las he- 
rramientas: SimMechanics y Virtual Reality Toolbox. La primera permite simular cuerpos en movimiento sin tener que analizar ninguna ecuación diferencial y la segunda permite visualizar e interactuar con sistemas dinámicos en un entorno de tres dimensiones; esto es una gran ventaja, ya que se puede observar en forma "real" el desempeño del robot y también la estética de la caminata.

El principal objetivo por el cual se realizó esta simulación es para determinar el tamaño del robot, es decir, se logró determinar los requerimientos de torque para los motores de ca- da articulación, que posteriormente se compararon con los valores de torques máximos de los servomotores disponibles en el mercado, limitando así el tamaño del robot.

También se probaron algunos algoritmos de monitorización y control en el "robot virtual", sin embargo, el requerimiento computacional necesario en el computador es un gran limitante, pudiendo tener simulaciones muy lentas.

La simulación dinámica del robot en MATLAB constituyó un aporte invaluable para esta tesis, ya que se probaron la mayoría de teorías y fun-

Foto 3

Simulación computarizada del robot

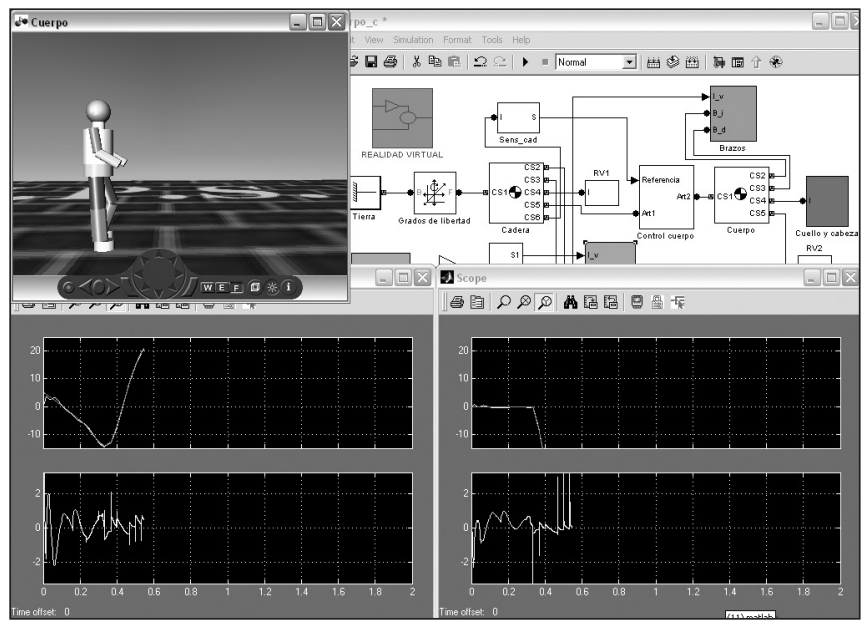


damentos de la caminata bípeda. Se ha podido determinar la gran importancia de una simulación en proyectos de este tipo, pues el investigador puede probar una serie de hipótesis sobre el fenómeno que estudia, sin que esto represente ningún riesgo ni desperdicio de recursos; es así, que se ha trabajado durante meses en el análisis físico y planeación de caminata previa a la construcción del robot. Se puede decir con propiedad que la simulación computarizada no es un aspecto opcional, representa un elemento fundamental en este tipo de proyectos.

\section{Descripción del proyecto}

El robot está compuesto básicamente de tres componentes: controlador (computador personal), módulo electrónico y estructura mecánica.

\section{a) Sistema mecánico}

Uno de los principales retos que plantea la construcción del robot bípedo es el diseño y elaboración del sistema mecánico, el mismo que debe cumplir con todos los requerimientos tanto en precisión, eficiencia, operatividad y principalmente desempeño.

Foto 4

Diagrama estructural del robot

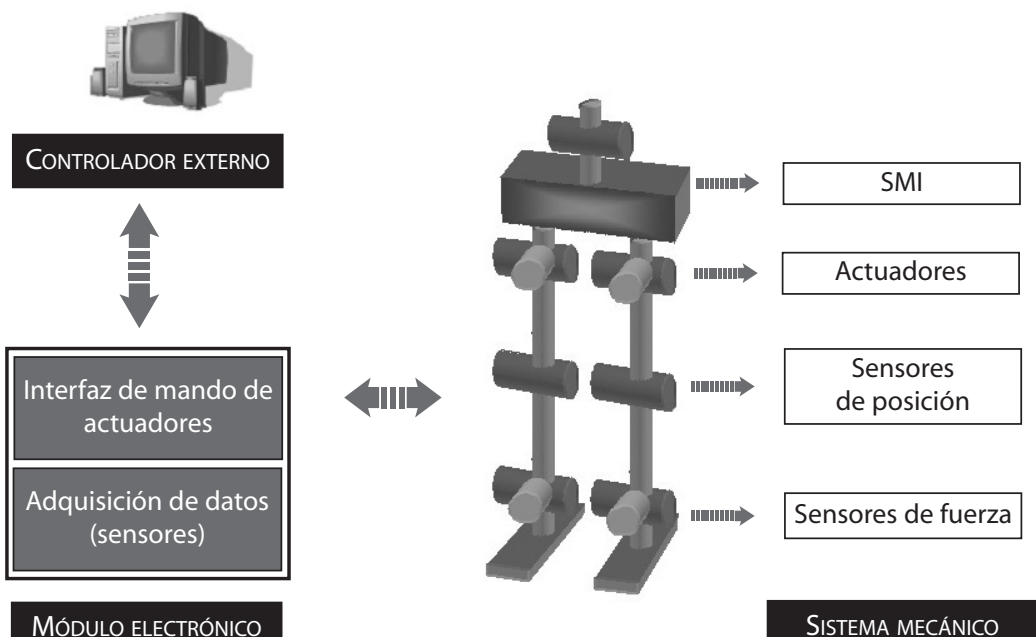

Módulo ELECTRÓNICO

SISTEMA MECÁNICO 
Fue de vital importancia en el diseño y construcción de un robot caminador tomar en cuenta todos los posibles movimientos que éste debe tener y cómo se relacionan mutuamente, con ello, se pone de manifiesto que en un ciclo de caminata normal todos los actuadores (motores) del robot van a estar en funcionamiento, lo cual plantea una gran complejidad del sistema mecánico.

Antes de iniciar la construcción del robot se realizó un profundo análisis estático y dinámico con el propósito de tomar en cuenta todos los efectos contraproducentes al movimiento de cada una de las partes del robot.

Manteniendo claros estos conceptos, el robot fue dotado con cinco Grados de Libertad (articulaciones simples giratorias) en cada pierna, que son justamente los principales movimientos que puede ejecutar el cuerpo humano para caminar en línea recta, de esta forma se puede generar movimientos bastante complejos y muy similares a los de la caminata humana.

Una de las partes fundamentales en la construcción del robot bípedo es la elección o el dimensionamiento de la estructura, la elección de estas medidas no fue tarea fácil, pues existían grandes limitantes como: el costo, limitantes de los motores, tamaño de los elementos, consumo de energía, etc., que representan grandes problemas y complicaciones tanto en la parte electrónica como mecánica.

Tras un breve análisis se puede observar que si se diseña un robot de tamaño natural, la construcción de las partes mecánicas se simplifica, ya que se cuenta con un gran espacio en el cual se puede trabajar y ubicar fácilmente no solamente los actuadores y sensores, sino además todas las placas electrónicas y demás elementos. Por otro lado, el gran tamaño del robot obliga a utilizar motores grandes donde la respuesta será lo suficientemente rápida para poder operar y efectuar los movimientos; además, el costo y la demanda de energía que se necesitaría para poder generar la caminata serían elevados.

Teniendo presente todo ello y algunas consideraciones más, se optó por construir un robot cuyas dimensiones giren alrededor de $50 \mathrm{~cm}$ de altura. Para cumplir con esta propuesta se tomaron en cuenta las dimensiones reales de una persona multiplicada por un factor de escalamiento cuyos resultados brindan las dimensiones reales de cada una de las partes.

Una vez recopilada toda la información acerca de los actuadores, sensores, tamaños de los elementos mecánicos, grados de libertad y cir- 


\section{Foto 5}

Diseño de una pieza en 2D

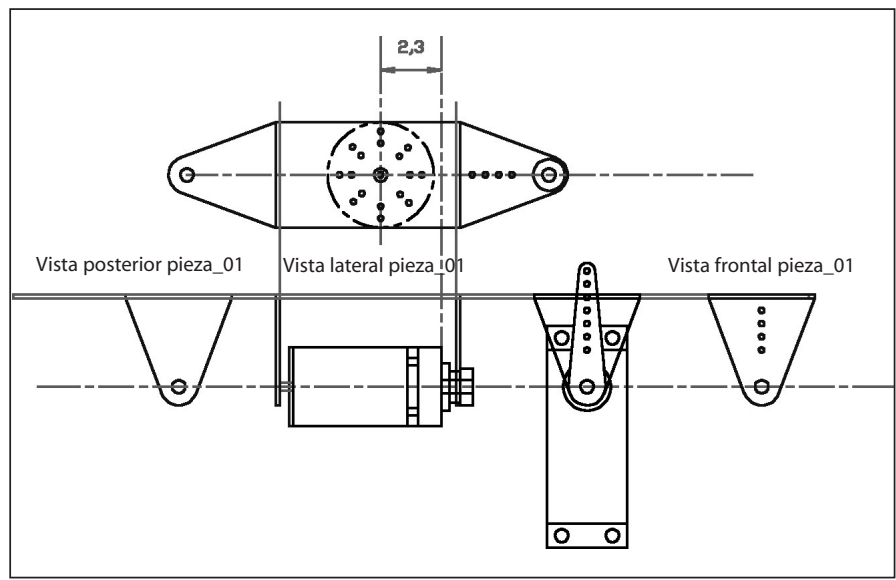

cuitos electrónicos, se procedió a diseñar en AUTOCAD cada una de las piezas mecánicas.

En el robot se distinguen básicamente dos tipos de sensores: Sensor de Medida Inercial (SMI) y Sensores de presión.

Los Sensores de Presión fueron ubicados en cada una de las plantas de los pies (4 en cada pie), los mismos recogen información sobre las fuerzas que interactúan entre el robot y el piso durante la caminata.

El Sensor de Medida Inercial ha sido instalado en el tronco del robot bípedo, que es el punto de referencia para los diferentes análisis y procedi- mientos. Este sensor se encarga de registrar aceleraciones lineales y velocidades angulares, las mismas que permiten, entre otras cosas, encontrar la inclinación absoluta del robot; información importante al momento de controlar el equilibrio del mismo.

\section{b) Módulo electrónico}

El módulo electrónico actúa como una interfaz entre el controlador externo y el sistema mecánico. Básicamente las funciones de este módulo son tres:

- Comandar y coordinar eficientemente el funcionamiento de 
los diferentes actuadores, los mismos que posteriormente definirán cada uno de los grados de libertad del robot bípedo, a partir de la información de posición enviada por el controlador externo.

- Chequear constantemente el estado de los diferentes sensores y enviar dicha información en forma rápida y segura hacia el controlador externo.

- Garantizar la adquisición de señales libres de ruido mediante el uso de adecuados sistemas de filtrado.

\section{c) Controlador Externo}

A partir de la información proporcionada por el sistema electrónico y parámetros proporcionados por el programador, el controlador se encarga de realizar los diferentes algoritmos y procedimientos necesarios para lograr la caminata bípeda. En esta ocasión el controlador ha sido implementado en un computador personal. En el siguiente diagrama funcional se explica en forma detallada las tareas del controlador.

Foto 6

Diagrama funcional del controlador

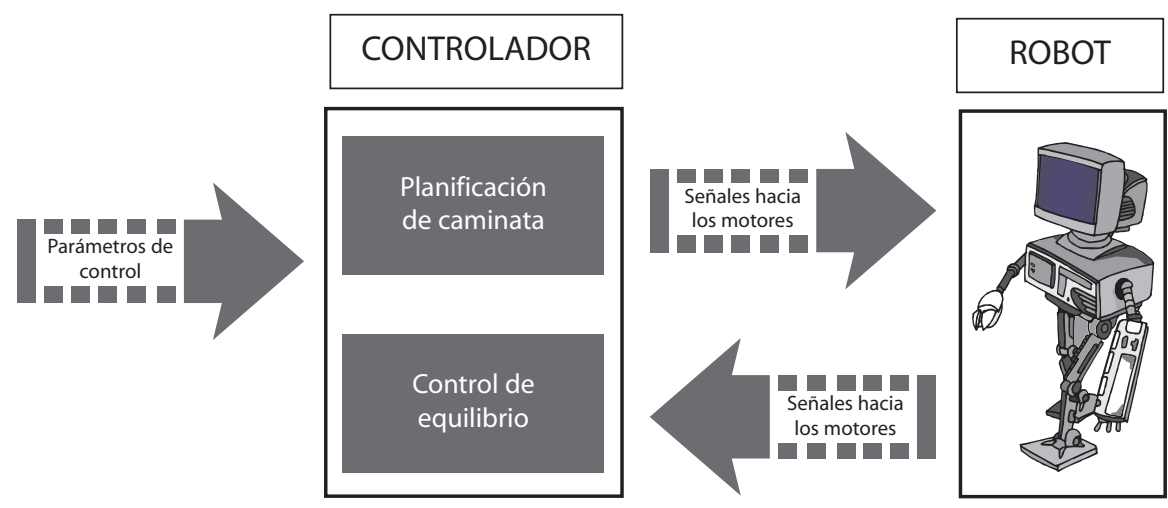


El bloque de planificación de caminata genera las diferentes secuencias y trayectorias que permiten que el robot camine.

Antes de explicar el bloque de control de equilibrio, es importante familiarizar al lector con algunos términos utilizados comúnmente en el estudio de la caminata bípeda:

- Polígono de soporte.- Es la figura geométrica plana formada en el piso por uno o dos pies, dependiendo del estado de la caminata.

- FRI (Foot Rotation Indicator Indicador de rotación del pie)."Es el punto en la superficie (dentro o fuera del polígono de soporte), en el cual la fuerza de reacción del piso tendría que actuar para que el pie de apoyo permanezca estacionario". Este punto ha sido determinado con el único objetivo de simplificar el análisis de la caminata bípeda, ya que si este punto está fuera del polígono de soporte el robot tiende a caer, es decir, que si este punto está siempre dentro del polígono de soporte, se garantiza la estabilidad dinámica de la caminata. La posición del FRI es una consecuencia directa del estado dinámico del robot. Algu-

Foto 7

Interfaz gráfica

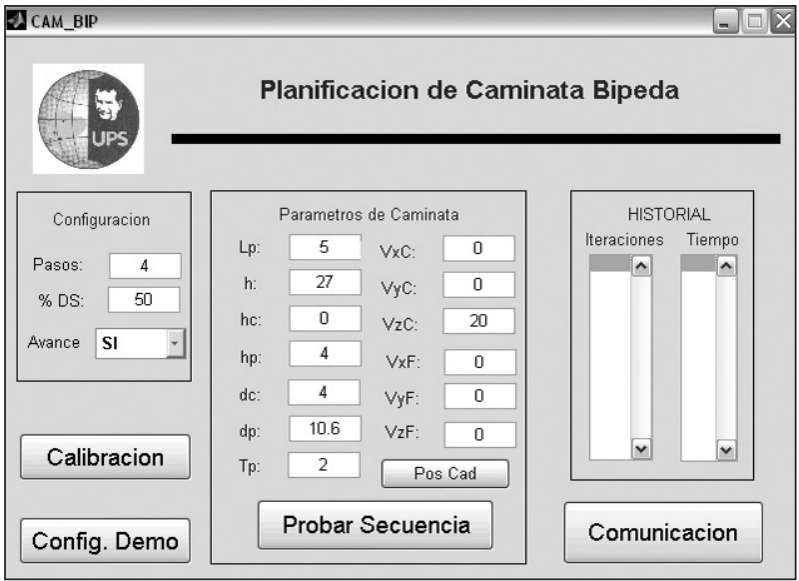


nos autores conocen a este punto como ZMP (Zero moment point - Punto de momentos cero). Entonces, la principal función del sistema de control de equilibrio es mantener al FRI dentro del polígono de soporte, para esto, debe realizar las correcciones necesarias en los movimientos del robot.

Finalmente, los parámetros de control son ingresados a través de una interfaz gráfica creada en MATLAB, la misma que permite configurar la caminata del robot. Entre los parámetros que se pueden ingresar se tiene: número de pasos, velocidad de caminata, geometría de la caminata, etc.

Resumiendo, entre las tareas del controlador se encuentran: manejo del flujo de datos, interpretación de los parámetros de control, simulación de los movimientos del robot, cálculo de la cinemática, dinámica (p. ej., de la ubicación del FRI), planificación de trayectorias, etc.

\section{Investigación a futuro}

A continuación se exponen algunos de los posibles temas que complementarían este proyecto, a fin de lograr una emulación mucho más robusta del comportamiento humano.
- Implementación del giro.- Actualmente el robot puede caminar en línea recta, hecho que limita su movilidad. Para lograr que el robot realice giros se deben añadir las articulaciones que permitan realizar este cometido; así como también, el análisis y desarrollo de la planificación de dichos giros. Evidentemente, si el robot tiene la capacidad de girar, éste puede dirigirse a cualquier lugar dentro de una superficie regular.

- Implementación y control de brazos.- Quizás este sea el aspecto determinante y la diferencia entre un robot bípedo y un humanoide. El robot al poseer brazos podría tomar y manipular objetos (ayudado por un sistema de visión), a más que los brazos en determinadas circunstancias ayudarían a mantener el equilibrio en la caminata, tal como ocurre en las personas. El hecho de añadir brazos al robot implica una mayor complejidad en la mecánica, análisis y control del robot.

- Sistema de audición.- Dentro de este sistema podemos destacar el reconocimiento de palabras y frases, la determinación del origen de un determinado sonido, identificación del hablante, etc., cualidades perfectamente dominadas por los seres humanos, pero una tarea ex- 
tremadamente ardua y complicada para las máquinas. Mediante la implementación de este sistema se crea la primera interfaz "amigable" hombre-robot, ya que se establece la interacción mediante el lenguaje. El robot podría reconocer ciertos comandos y órdenes, que inmediatamente serían ejecutadas conforme a las capacidades del robot.

- Sistema de visión.- Permitirán al robot conducirse a través de ambientes con la presencia de obstá- culos, tales como muros, pilares y demás, para esto se debe analizar e implementar algoritmos de visión estereoscópica (percepción de la profundidad - visión tridimensional). Se encuentran ya en desarrollo los algoritmos para la detección de objetos móviles, los cuales permitirán que el robot identifique objetivos dentro de un ambiente hacia los cuales dirigirse sin necesidad de ser especificados por un agente externo como es el programador.

\section{Foto 8}

Esquema general del sistema de visión estereoscópica

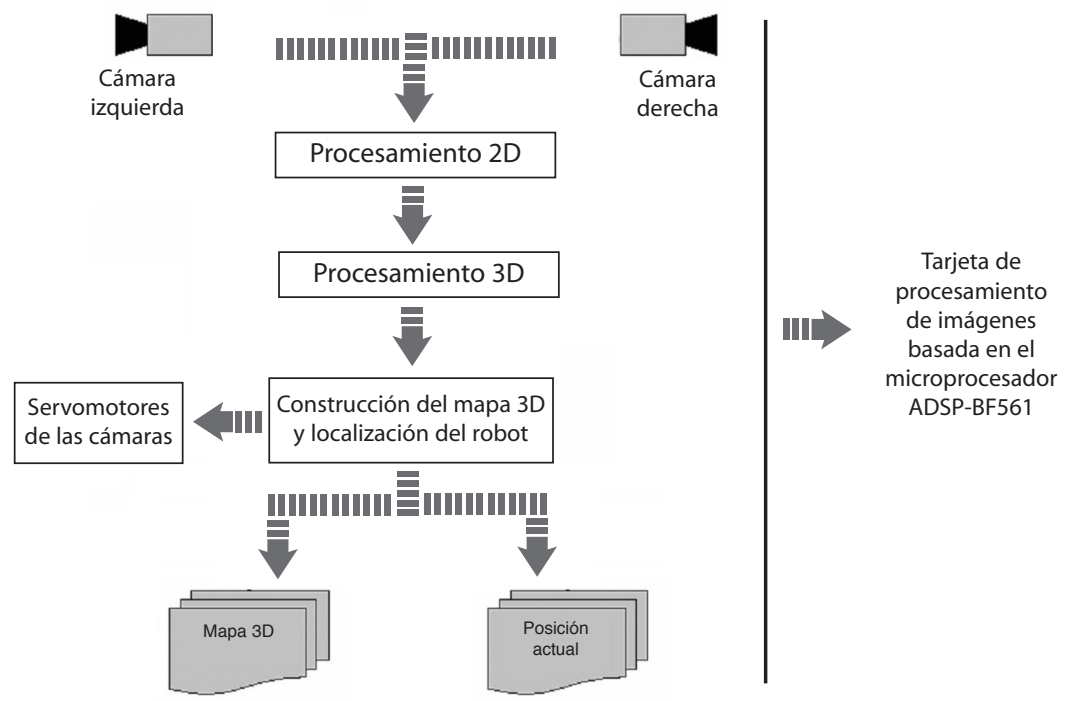


- Desarrollo de Inteligencia.- Entre otras cosas, el robot deberá tener la capacidad de tomar decisiones adecuadas ante situaciones inesperadas. Otro aspecto a considerar es el autoaprendizaje, que por el momento es una característica inherente a los humanos; es decir, que mientras mayor atención y enseñanza reciba el robot, mayores serán sus capacidades y logros.

Son innumerables las posibilidades de desarrollo que ofrece esta temática, de hecho, se conoce de grandes avances dentro de la emulación de los sentidos, hablamos de la interpretación del olor y sabor, y por supuesto sensores de tacto. Robots que cada vez más se aproximan a los humanos, en cuanto a su desempeño y apariencia, desarrollo limitado únicamente por la velocidad de los procesadores de la actualidad, situación que seguramente no durará mucho tiempo.

\section{Conclusiones}

El desarrollo de un robot bípedo, al ser un tema de investigación relativamente nuevo, requiere de un exhaustivo estudio, en donde la física, matemáticas, teoría de control, etc., se juntan para intentar establecer principios y teoremas generales que describan el comportamiento de la caminata bípeda. Muchos e importantes aportes han sido realizados por científicos en todo el mundo, sin embargo, todavía existe una gran diferencia entre la caminata natural humana y la caminata artificial robotizada, tanto en estética como en eficiencia, es decir, que todavía queda mucho trabajo que hacer si el objetivo es emular el modo de caminar de las personas.

Quizás el objetivo principal por el cual se realizó este proyecto fue el de IMPULSAR y COMENZAR con este tipo de intentos sobre todo en nuestro medio, objetivo que ha sido cumplido, pues queda sentada ya la base teórica, material en extremo importante sobre el cual pueden surgir proyectos de investigación de mayor jerarquía, incluso en temas complementarios, ya que los principios de la caminata bípeda bien podrían ser aplicados en el diseño y construcción de prótesis o en el estudio de deportistas de alto rendimiento.

"Un robot capaz de movilizarse en dos piernas!" para algunos una utopía (en nuestro medio obviamente), para otros no tiene sentido y la mayoría pregunta "Qué más hace el robot?, El robot sólo camina?, etc."; para los autores de este proyecto simplemente representó una gran oportunidad para investigar, una maravillosa oportunidad para aprender. 
José Larriva, Edyson Guillermo, José Trelles, Oscar Vele

\section{Foto 9}

Página WEB del proyecto

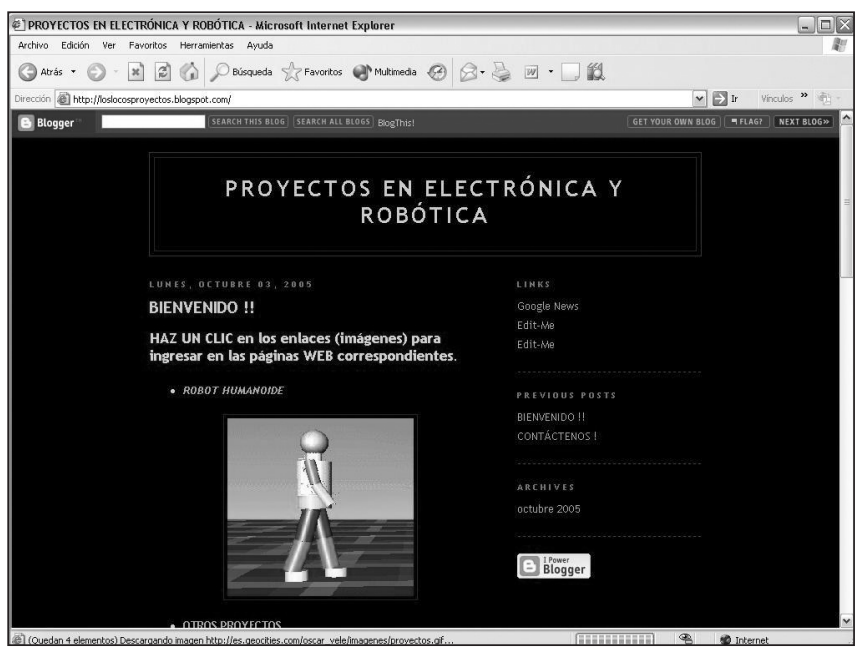

\section{Foto 10}

Equipo de trabajo del proyecto

De izquierda a derecha:Tnlg. José Trelles, Tnlg. Oscar Vele, Tnlg. Edyson Guillermo, Tnlg. José Larriva.

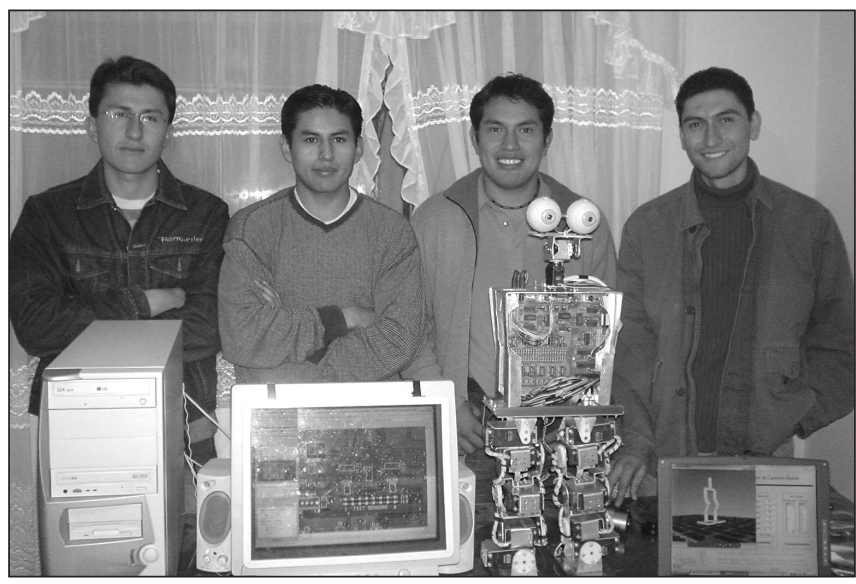


Este proyecto ha sido desarrollado en dos tesis, cada una con un respetable nivel de complejidad, además de ser dos estudios que se complementan para lograr un objetivo común. Dos tesis en las que la una no es más importante que la otra, ya que del éxito de cada una de ellas depende la superación del proyecto. Una de las tesis se ocupó del estudio, análisis y control de la caminata bípeda, lo que viene a ser el desarrollo teórico del proyecto. La tesis complementaria se encargó de la realización del sistema mecatrónico sobre el cual se probaron con éxito los algoritmos y sistemas propuestos.

Es indudable que existe mucho trabajo que se deja pendiente dentro del desarrollo de un robot bípedo, quizás eso justifique los 20 años y millones de dólares que la firma Honda ha invertido en la realización de sus robots humanoides.

Una página WEB para difundir los conocimientos adquiridos duran- te la realización de este proyecto ha complementado nuestro trabajo. Es fundamental concienciar a los estudiantes y nuevos investigadores que un conocimiento debe ser difundido de forma adecuada ya que es la única manera que el nivel científico y tecnológico en nuestro medio se desarrolle; y para cumplir con este cometido también se requiere de un mayor apoyo institucional y económico a los proyectos investigativos; sin embargo, la astucia y perseverancia de los estudiantes siempre serán la piedra angular de cualquier GRAN HAZAÑA TECNOLÓGICA.

\section{Información}

Para mayor información del proyecto visite:

http://loslocosproyectos.blogspot.com

o contáctenos a: loslocosproyectos@yahoo.com 